\title{
GENERIC AUTOMORPHISMS WITH PRESCRIBED FIXED FIELDS
}

\author{
Bijan Afshordel
}

September 10, 2010

\begin{abstract}
This article addresses the question which structures occur as fixed structures of stable structures with a generic automorphism. Among others we give a Galois theoretic characterisation. As application we prove: Any pseudofinite field is the fixed field of some model of $A C F A$. Any one-free pseudo-differentially closed field of characteristic zero is the fixed field of some model of $D C F A$. Any one-free PAC field of finite degree of imperfection is the fixed field of some model of $S C F A$
\end{abstract}

\section{Introduction}

A difference field is a field with a distinguished automorphism. They were studied by model theorists since the 1990's, while originating in the work of Birkhoff, Ritt, Cohn and others in the early 20th century. A difference field is called generic, and its underlying automorphism is called a generic automorphism, if it is existentially closed in all its difference field extensions. The term difference closed field is used as a synonyme. It is well-known that the class of generic difference fields is elementary, its theory being called $A C F A$.

The fixed field of a generic automorphism is a pure pseudofinite field, and given a pseudofinite field $k$ one can fairly easily show that there is some model of $A C F A$ whose fixed field is elementarily equivalent to $k$.

We ask whether, given a pseudofinite field $k$, is there a model of $A C F A$ whose fixed field actually equals $k$. Note that in characteristic zero any generic difference field has infinite transcendence degree, while there are plenty pseudofinite subfields of the algebraic closure of $\mathbb{Q}$.

The same question arises in the context of difference-differential fields of characteristic zero, whose common theory admits a model companion called $D C F A$, and in the context of fields with a finite named $p$-basis endowed with an automorphism fixing the $p$-basis, whose common theory also admits a model companion that we call $S C F A$.

We give a positive answer to each of the above questions by passing to the following more general context. Fix a countable stable $L$-theory $T$ with elimination 
of imaginaries and quantifier elimination. Let $\sigma$ be a new function symbol and $L_{\sigma}=L \cup\{\sigma\}$. We denote $T_{\sigma}$ the theory whose models are $L_{\sigma}$-structures $(M, \sigma)$, where $M \models T$ and $\sigma \in \operatorname{Aut}(M)$ is an automorphism of $M$. We assume that $T_{\sigma}$ has a model companion, which we call $T A$, and that $T A$ has elimination of imaginaries. Given a model $(M, \sigma)$ of $T A$ we write $\operatorname{Fix}(M, \sigma)$ for the subset of $M$ which is fixed by $\sigma$ pointwise, which is naturally an $L$-substructure of $M$. If $A$ is any subset of some model $M$ of $T$, we denote by $\operatorname{Gal}(A)$ the group of permutations of the algebraic closure of $A$ in $M$ that leave $A$ pointwise fixed and are elementary in the sense of the $L$-structure $M$. It is a profinite group in a natural way and we call it the absolute Galois group of $A$. If $K$ is an $L$-structure elementarily equivalent to $\operatorname{Fix}(M, \sigma)$, we can embed $K$ into some model of $T$ and assigm to $K$ its absolute Galois group as a subset of that model. By virtue of quantifier elimination of $T$ this does not dependend on the embedding chosen, and so $\mathrm{Gal}(K)$ is an invariant of $K$ and we speak about the absolute Galois group of $K$. In this setting we prove:

Theorem 1.1 Let $(M, \sigma)$ be a model of $T A$ and $K \equiv_{L} \operatorname{Fix}(M, \sigma)$. Then there is some model $(N, \sigma)$ of $T A$ with $\operatorname{Fix}(N, \sigma)=K$ if and only if $\operatorname{Gal}(K)=\widehat{\mathbb{Z}}$, the profinite completion of $\mathbb{Z}$.

We note that the only if part should be folklore. In the context of fields we obtain the following corollaries.

Corollary 1.2 Any difference field whose fixed field $k$ is pseudofinite embeds into some model of ACF A with fixed field $k$.

Corollary 1.3 Any difference-differential field of charactieristic zero whose fixed differential field $(k, d)$ is one-free pseudo-differentially closed embeds into some model of DCFA with fixed differential field $(k, d)$.

Corollary 1.4 Any difference field whose fixed field $k$ is one-free PAC and of finite Ershov invariant embeds into some model of SCF A with fixed field $k$.

We are not going to recall but use freely stability and simplicity theory. Paragraph 2 introduces notation and provides preliminaries about stable structures with a generic automorohism and their fixed structures. In paragraph 3 we introduce the notion of conservative embedding over a subset, see below. We show that if $K \preccurlyeq_{L} \operatorname{Fix}(M, \sigma \phi)$ and if $K$ and $\operatorname{Fix}(M, \sigma)$ satisfy a certain condition on definable and algebraic closures, then $\operatorname{Fix}(M, \sigma)$ is conservatively embedded over $K$ in $(M, \sigma)$ for any model $(M, \sigma)$ of $T A$. This is used in paragraph 4 in the proof of theorem (1.1), where we also discuss some variants. The applications to generic automorphisms of various theories of fields are dealt with in section 5. The last paragraph is independent from the rest of the article and contains a result concerning elimination of imaginaries.

Acknowledgements: We would like to express our gratitude to Martin Ziegler for his most valuable comments. We also thank Immanuel Halupcok, Richard Elwes and Mihai Prunescu for helpful discussions. Special thanks are due to Olivier A. Roche for the valuable discussions which made this work possible. Last but not least we would like to mention that Zoé Chatzidakis has proved corollary 1 independently in yet unpublished work. We are deeply indebted to her for her encouragement to go further in this direction. 


\section{Preliminaries}

This section introduces notation and recalls some known facts about stable theories with a generic automorphism. Our main reference is [6].

Let $T$ be a be a complete stable theory with infinite models and with elimination of imaginaries and quantifier elimination in the language $L . \quad \operatorname{acl}_{\mathrm{T}}$ and $\mathrm{dcl}_{\mathrm{T}}$ denote the algebraic resp. definable closure in models of $T$. For a subset $A \subset M$ of some model of $T$ we write $\operatorname{Gal}(A)$ for the group of elementary permutations of $\operatorname{acl}_{\mathrm{T}}(A)$ that leave $A$ pointwise fixed and call it the Galois group of $A$. For a tuple $\bar{a}$, not necessarily finite, and a parameter set $A$ we denote by $\operatorname{tp}_{T}(\bar{a} / A)$ the type of $\bar{a}$ over $A$ and $\operatorname{qftp}_{T}(\bar{a} / A)$ for the quantifier-free type of $\bar{a}$ over $A$.

We let $L_{\sigma}=L \cup\{\sigma\}$, where $\sigma$ is a new unary function symbol, and $T_{\sigma}$ be the theory whose models are those $L_{\sigma}$-structures $(M, \sigma)$ with $M \models T$ and $\sigma \in \operatorname{Aut}(M)$. By abuse of notation we write $\sigma$ for the function symbol as well as for the automorphism of $(M, \sigma)$. We always assume that $T_{\sigma}$ has a model companion, which we denote by $T A$, and say $T A$ exists for short. So in particular $T A$ is model complete. We write $\operatorname{acl}_{\sigma}$ and $\mathrm{dcl}_{\sigma}$ for the algebraic resp. definable closure in models of $T A$. If $A$ is a subset of some model of $T A$ then $\operatorname{cl}_{\sigma}(A)$ denotes the closure of $A$ under $\sigma$ and $\sigma^{-1}$. Of course these closures depend on the model of $T A$ they are taken in, however there will be no ambiguity so that we use this simple notation. Also, if $\bar{a}$ is a tuple in some model of $T A$ and $A$ is a parameter set, we write $\operatorname{tp}_{\sigma}(\bar{a} / A)$ for the type of $\bar{a}$ over $A$ and $\operatorname{qftp}_{\sigma}(\bar{a} / A)$ for the quantifier-free type of $\bar{a}$ over $A$, both in that model.

For any $L_{\sigma}$-structure $(A, \alpha)$, not necessarily a model of $T_{\sigma}$, we denote by

$$
\operatorname{Fix}(A, \alpha)=\{x \in A \mid \alpha(x)=x\}
$$

the set of elements of $A$ which are invariant under $\alpha$. By remark (2.1) below, $\operatorname{Fix}(M, \sigma)$ is an $L$-substructure of $M$ for any model $(M, \sigma)$ of $T A$; we will not distinguish between the subset $\operatorname{Fix}(M, \sigma)$ of $M$ and the $L$-substructure $\operatorname{Fix}(M, \sigma)$ of $M$, and talk about the fixed structure of $(M, \sigma)$.

Let us observe a set of basic facts, which we state in remarks for future reference.

Remark 2.1 Let $T$ be as above and $(M, \sigma)$ be a model of $T_{\sigma}$. Let further $\phi$ be an automorphism of $M$ that is $L$-definable without parameters.

1. $(M, \sigma)$ is a model of $T A$ if and only if $(M, \sigma \phi)$ is, and moreover a subset of $M$ is definable in the $L_{\sigma}$-structure $(M, \sigma)$ using parameters from $A \subset M$ if and only if it is A-definable in the $L_{\sigma}$-structure $(M, \sigma \phi)$.

2. $\operatorname{Fix}(M, \sigma)$ is a $\mathrm{dcl}_{\mathrm{T}}$-closed subset of $M$. If $(M, \sigma)$ is a model of TA, then $\operatorname{Fix}(M, \sigma)$ is non-empty, and hence we can view it as an L-substructure of $M$. The same applies to $\operatorname{Fix}(M, \sigma \phi)$.

Proof. We only prove that $\operatorname{Fix}(M, \sigma) \neq \emptyset$ in case $(M, \sigma)$ is a model of $T A$ and leave the rest to the reader. So let $(M, \sigma) \models T A$. Choose an element $a \notin M$ in some elementary extension of $M$. Then $\operatorname{tp}_{T}(a / M)$ is stationary because $T$ is stable. Therefore $\sigma\left(\operatorname{tp}_{T}(a / M)\right)=\operatorname{tp}_{T}(a / M)$, whence the map $\sigma \cup\{(a, a)\}$ is elementary in the sense of $T$, and we find a model $(N, \sigma)$ of $T_{\sigma}$ that extends 
$(M, \sigma)$ and contains $a$, and with $\sigma(a)=a$. But $(M, \sigma)$ is existentially closed in $(N, \sigma)$ as $T A$ is the model companion of $T_{\sigma}$, so the assertion follows.

Remark 2.2 Let $T$ be as above and $(M, \sigma)$ be a model of $T A$. Then every automorphism of the $L$-structure $\operatorname{Fix}(M, \sigma)$ is elementary in the sense of $T$.

Proof. This is valid even if $(M, \sigma)$ is only a model of $T_{\sigma}$ and follows from quantifier elimination of $T$.

Remark 2.3 Let $T$ be as above. Then $\operatorname{Gal}(\operatorname{Fix}(M, \sigma))=\widehat{\mathbb{Z}}$ for any model $(M, \sigma)$ of $T A$.

Proof. Again we believe this is folklore, but for lack of reference we sketch a possible proof. As $\operatorname{Fix}(M, \sigma)=\operatorname{Fix}\left(\operatorname{acl}_{\mathrm{T}}(\operatorname{Fix}(M, \sigma)), \sigma\right)$, it follows from Galois theory [14] that $\operatorname{Gal}(\operatorname{Fix}(M, \sigma))$ is procyclic. So we only have to show that for any $n<\omega$ there is some tuple $a$ with exactly $n$ conjugates over $\operatorname{Fix}(M, \sigma)$. Let to that end $a_{0}, \ldots, a_{n-1}$ be the beginning of a Morley sequence over $M$ and define a map $\tau$ on $M \cup\left\{a_{0}, \ldots, a_{n-1}\right\}$ to be $\sigma$ on $M, \tau\left(a_{n-1}\right)=a_{0}$ and $\tau\left(a_{\nu}\right)=a_{\nu+1}$ otherwise. As $\left\{a_{0}, \ldots, a_{n-1}\right\}$ is an indiscernible set it follows that $\tau$ is elementary in the sense of $T$, so as in the proof of remark (2.1) we conclude that there is $a \in M$ with exactly $n$ conjugates over $\operatorname{Fix}(M, \sigma)$.

The following fact was proved by Chatzidakis and Pillay in [6].

Fact 2.4 (Chatzidakis-Pillay) Let $T$ be a complete stable L-theory with quantifier elimination and elimination of imaginaries such that TA exists. Then the following hold.

1. Let $\left(M_{1}, \sigma_{1}\right)$ and $\left(M_{2}, \sigma_{2}\right)$ be models of $T A$ containing a common substructure $(A, \sigma)$, and let $A$ be $\operatorname{acl}_{\mathrm{T}}$-closed. Then $\left(M_{1}, \sigma_{1}\right) \equiv_{A}\left(M_{2}, \sigma_{2}\right)$. In particular any bijection between acl $_{\sigma}$-closed sets that is elementary in the sense of $T$ and commutes with $\sigma$ is elementary in the sense of TA. See [6], proposition (3.5.2).

2. If $A$ is a subset of a model of $T A$, then $\operatorname{acl}_{\sigma}(A)=\operatorname{acl}_{\mathrm{T}}\left(\operatorname{cl}_{\sigma}(A)\right)$, see [6], lemma (3.6).

3. For subsets $A, B$ and $C$ of a model of $T A, A$ is said to be independent from $C$ over $B$ if and only if $\operatorname{acl}_{\sigma}(A)$ is independent from $\operatorname{acl}_{\sigma}(C)$ over $\operatorname{acl}_{\sigma}(B)$ in the sense of $T$. This is non-forking independence and turns TA into a simple theory. See [6], corollary (3.8).

Quantifier-free Stability. We will later be interested in fields with a generic automorphism. In these cases, $T A$ is simple unstable. However, the quantifierfree fragment of $T A$ is always stable by the following lemma. Recall that a first oder theory $T^{\prime}$ is quantifier-free stable if for all cardinals $\lambda$ with $\lambda^{\left|T^{\prime}\right|}=\lambda$ and any parameter set $A$ of size $\lambda$ there are at most $\lambda$ quantifier-free types over $A$. Equivalently, every quantifier-free formula has finite $\Delta$-rank for all finite sets $\Delta$ of quantifier-free formulae. 
Lemma 2.5 Let $T$ be a stable complete L-theory with quantifier elimination and elimination of imaginaries such that $T A$ exists. Then $T A$ is quantifier-free stable (i.e. every completion of $T A$ is quantifier-free stable). Furthermore, if $T$ is totally transcendental then $T A$ is quantifier-free totally transcendental.

Proof. Let $\sigma \in \operatorname{Aut}_{T}(\mathfrak{C})$ such that $(\mathfrak{C}, \sigma) \models T A$ and $A$ be a subset of $(\mathfrak{C}, \sigma)$ of size $\lambda$ with $\lambda^{|T|}=\lambda$. Then $\lambda^{\omega}=\lambda$. We may assume that $\mathrm{A}$ is acl $\sigma_{\sigma}$-closed . Then $A$ is also acl ${ }_{\mathrm{T}}$-closed. Consider a tuple $\bar{a} \in(\bar{M}, \sigma)$. Obviously $\operatorname{qftp}_{\sigma}(\bar{a} / A)$ is uniquely determined by $\operatorname{qftp}_{T}\left(\sigma^{i}(\bar{a})_{i \in \mathbb{Z}} / A\right)$ by quantifier elimination of $T$. As $T$ is stable there are only $\lambda$-many (quantifier-free) types over $A$ of sequences of length $\omega$.

The second part was proved by Bustamante-Medina, see [2], the discussion preceding remark (3.32). His proof is for countable $T$, but clearly works for arbitrary cardinality of $T$.

So in particular we have local ranks on quantifier-free formulae in $T A$. Quantifierfree stability of $T A$ has the following consequence, which will play an important rôle in our proof of theorem (4.1). Recall that for a theory $T^{\prime}$ and parameter set $A$ in $T^{\prime}$ the locally isolated quantifier-free types are said to be dense in (the space of) quantifier-free types over $A$ if for any quantifier-free formula $\varphi(\bar{x})$ in the language of $T^{\prime}$ there is some quantifier-free type $\pi$ containing $\varphi$ such that for any finite set $\Delta$ of quantifier-free formulae there is some $\delta(\bar{x}) \in \pi$ (in the language of $\left.T^{\prime}\right)$ such that $\left.\delta(\bar{x}) \vdash \pi\right|_{\Delta}$.

Proposition 2.6 Let $T$ be a countable complete stable L-theory, with quantifier elimination and elimination of imaginaries. Assume that TA exists and eliminates imaginaries. Then for any completion of $T A$ and any parameter set A (in the sense of that completion) the locally isolated quantifier-free types are dense in the space of quantifier-free types over A. If T is totally transcendental, not necessarily countable, then the isolated quantifier-free types are dense in the space of quantifier-free types over A.

Proof. It is well-known that in a countable stable theory the locally isolated types are dense over any parameter set (see for example [16], theorem (11.8)). Even if $T A$ might be unstable, its quantifier-free fragment is stable by lemma (2.5). It is countable because $T$ is. From now on the proof follows the same line as for countable stable theories: Let $\varphi(\bar{x})$ be a quantifier-free $L_{\sigma}$-formula and $\left(\Delta_{i}\right)_{i \in \mathbb{N}}$ be an enumeration of all finite sets of quantifier-free $L_{\sigma}$-formulae $\psi(\bar{x}, \bar{y})$. One constructs recursively a sequence $\varphi_{i}(\bar{x})$ of quantifier-free $L_{\sigma}(A)$ formulae. Starting with $\varphi_{0}(\bar{x})=\varphi(\bar{x})$ one lets $\varphi_{n+1}(\bar{x})$ be a quantifier-free $L_{\sigma}(A)$-formula of minimal $\Delta_{n+1}$-rank and -degree with $\models \varphi_{n+1}(\bar{x}) \rightarrow \varphi_{n}(\bar{x})$. Then $\left\{\varphi_{n}(\bar{x}) \mid n \in \mathbb{N}\right\}$ axiomatises a locally isolated quantifier-free type over $A$ that contains $\varphi(\bar{x})$.

Stable embedding. Let $M$ be an $L^{\prime}$-structure, $L^{\prime}$ an arbitrary first order language, and $n \in \mathbb{N}$. Recall that a $\emptyset$-definable subset $P$ of $M^{n}$ is called stably embedded in $M$ if for any $m$ and any set $X \subset M^{m n}$ which is definable using parameters, $X \cap P^{m}$ is definable using parameters from $P$. Chatzidakis and Hrushovski prove the following lemma in [4]. 
Lemma 2.7 (Chatzidakis-Hrushovski) Let $M$ be sufficiently saturated and $P \subset M^{n}$ be definable over $\emptyset$. Then $P$ is stably embedded in $M$ if and only if any automorphism of $P^{\text {ind }}$ lifts to an automorphism of $M$. Here $P^{\text {ind }}$ denotes $P$ with its induced structure from $M$, i.e. the $\emptyset$-definable subsets of $P^{m}$ are those of the form $P^{m} \cap X$ for $\emptyset$-definable $X \subset M^{m n}$.

Remark 2.8 If $T A$ eliminates imaginaries, then $\operatorname{Fix}(M, \sigma)$ is stably embedded for all models $(M, \sigma)$, for the canonical parameter of $\sigma(x)=x$ is fixed by $\sigma$.

\section{Conservative Embedding}

Definition 3.1 Let $L_{1}$ and $L_{2}$ be first-order languages with $L_{1} \subset L_{2}$. Let furthermore $P$ be an $L_{1}$-substructure of the $L_{2}$-structure $M$ and $A$ be a subset of $P$. We say that $P$ is conservatively embedded over $A$ in $M$ if every subset of (some cartesian power of) $P$ that is A-definable in the $L_{2}$-structure $M$, is $A$-definable in the $L_{1}$-structure $P$.

Note that this is different from the notion of conservative extension of theories. Note also that in the special case that $L_{1}=L_{2}$ and $A=P$, if $P$ is $\emptyset$-definable and conservatively embedded over $A$ in $M$, then $P$ is stably embedded in $M$.

Our aim in the present section is to show in proposition (3.7) that if $T$ is a stable $L$-theory with quantifier elimintion and elimination of imaginaries such that $T A$ exists and eliminates imaginaries, and if $(M, \sigma)$ is a model of $T A$, then the fixed structure $\operatorname{Fix}(M, \sigma)$ is conservatively embedded over every $L$ elementary substructure $K$ such that

$$
\operatorname{dcl}_{\mathrm{T}}\left(\operatorname{acl}_{\mathrm{T}}(K), \operatorname{Fix}(M, \sigma)\right)=\operatorname{acl}_{\mathrm{T}}(\operatorname{Fix}(M, \sigma)) .
$$

It will turn out that this condition on definable and algebraic closures is also necessary, see theorem (4.1), but first of all we show that conservative embedding is preserved in elementary extensions in the following sense.

Lemma 3.2 Let $L_{1}$ and $L_{2}$ be first-order languages with $L_{1} \subset L_{2}$ and $M_{1} \preccurlyeq M_{2}$ be an elementary extension of $L_{2}$-structures. Let further $\varphi$ be an $L_{2}$-formula without parameters. Assume that $\varphi\left(M_{1}\right)$ is an $L_{1}$-substructue of $M_{1}$ and $A \subset$ $\varphi\left(M_{1}\right)$. Then $\varphi\left(M_{1}\right)$ is conservatively embedded in $M_{1}$ over $A$ if and only if $\varphi\left(M_{2}\right)$ is so in $M_{2}$.

Proof. The proof is straightforward using relativisation of quantifiers.

Also the above condition on definable and algebraic closures is preserved in elementary extensions.

Proposition 3.3 Let $T$ eliminate imaginaries and $(N, \sigma) \preccurlyeq(M, \sigma)$ be models of $T_{\sigma}$. Then $\operatorname{dcl}_{\mathrm{T}}\left(\operatorname{acl}_{\mathrm{T}}(\operatorname{Fix}(N, \sigma)), \operatorname{Fix}(M, \sigma)\right)=\operatorname{acl}_{\mathrm{T}}(\operatorname{Fix}(M, \sigma))$.

Proof. We denote $F_{M}=\operatorname{Fix}(M, \sigma)$ and $F_{N}=\operatorname{Fix}(N, \sigma)$. We will show that $\operatorname{Aut}_{T}\left(\operatorname{acl}_{\mathrm{T}}\left(F_{M}\right) / \operatorname{acl}_{\mathrm{T}}\left(F_{N}\right), F_{M}\right)$ is trivial. By Galois theory (see [14]) the automorphism $\sigma$ (of $M)$ restricts to topological generators of $\operatorname{Gal}\left(F_{M}\right)$ and $\operatorname{Gal}\left(F_{N}\right)$ 
respectively because $F_{M}=\operatorname{Fix}\left(\operatorname{acl}_{\mathrm{T}}\left(F_{M}\right), \sigma\right)$ and $F_{N}=\operatorname{Fix}\left(\operatorname{acl}_{\mathrm{T}}\left(F_{N}\right), \sigma\right)$. Hence both $\operatorname{Gal}\left(F_{M}\right)$ and $\operatorname{Gal}\left(F_{N}\right)$ are procyclic and the natural restriction map

$$
\operatorname{Gal}\left(F_{M}\right) \stackrel{\text { res }}{\longrightarrow} \operatorname{Gal}\left(F_{N}\right)
$$

is surjective. Thus $\operatorname{Aut}_{T}\left(\operatorname{acl}_{\mathrm{T}}\left(F_{M}\right) / \operatorname{acl}_{\mathrm{T}}\left(F_{N}\right), F_{M}\right)$ is trivial if and only if res is an isomorphism. Again by Galois theory (see [14]) it suffices to show that for all $n \in \mathbb{N}$ and $\bar{b} \in \operatorname{acl}_{\mathrm{T}}\left(F_{M}\right)$ of degree $n$ over $F_{M}$ there is $\bar{c} \in \operatorname{acl}_{\mathrm{T}}\left(F_{N}\right)$ of degree $n$ over $F_{N}$.

To see this, note that as $\sigma$ generates $\operatorname{Gal}\left(F_{M}\right)$ and $\bar{b}$ has degree $n$ over $F_{M}$, we have

$$
(M, \sigma) \models \exists \bar{x} \quad \sigma^{n}(\bar{x})=\bar{x} \wedge \bigwedge_{i=1}^{n-1} \sigma^{i}(\bar{x}) \neq \bar{x}
$$

and thus

$$
(N, \sigma) \models \exists \bar{x} \quad \sigma^{n}(\bar{x})=\bar{x} \wedge \bigwedge_{i=1}^{n-1} \sigma^{i}(\bar{x}) \neq \bar{x},
$$

so we are done.

The next two lemmata are easy observations.

Lemma 3.4 Let $T$ have quantifier elimination. Then for any model $(M, \sigma)$ of $T_{\sigma}$ and $K \preccurlyeq_{L} \operatorname{Fix}(M, \sigma)$ we have $\operatorname{acl}_{\mathrm{T}}(K) \cap \operatorname{Fix}(M, \sigma)=K$.

Proof. The proof is straightforward using quantifier elimination of $T$.

Lemma 3.5 Let $T$ be a stable theory with elimination of imaginaries, $A$ a set of parameters, and $p \in S\left(\operatorname{acl}_{\mathrm{T}}(A)\right)$. If $\beta \in \operatorname{Aut}(\mathbb{C} / A)$ is an automorphism of the monster model of $T$ over $A$ such that $\beta(p)=p$, then $\left.p\right|_{\operatorname{acl}(A) \cap \operatorname{Fix}(\beta)}$ is stationary.

Proof. By elimination of imaginaries the canonical base $c$ of $p$ is contained in $\operatorname{acl}_{\mathrm{T}}(A)$. As $\beta(p)=p$, it follows that $c$ is also contained in $\operatorname{Fix}(\beta)$.

Lemma 3.6 Let $T$ be a stable L-theory with quantifier elimination and elimination of imaginaries such that $T A$ exists and eliminates imaginaries. Let $(M, \sigma)$ be a sufficiently saturated model of $T A$ and $K \preccurlyeq_{L} \operatorname{Fix}(M, \sigma)$. If

$$
\operatorname{dcl}_{\mathrm{T}}\left(\operatorname{acl}_{\mathrm{T}}(K), \operatorname{Fix}(M, \sigma)\right)=\operatorname{acl}_{\mathrm{T}}(\operatorname{Fix}(M, \sigma))
$$

then the natural restriction map

$$
\text { res : } \operatorname{Aut}_{L_{\sigma}}((M, \sigma) / K) \longrightarrow \operatorname{Aut}_{L}(\operatorname{Fix}(M, \sigma) / K)
$$

is surjective. Here $\operatorname{Aut}_{L}(\operatorname{Fix}(M, \sigma) / K)$ denotes the group of automorphisms over $K$ of the L-structure $\operatorname{Fix}(M, \sigma)$.

Note that if $T$ and $T A$ are as in lemma (3.6) and if $\phi$ is an automorphism of $T$ that is $L$-definable over $\emptyset$, and if $K \preccurlyeq_{L} \operatorname{Fix}(M, \sigma \phi)$ with

$$
\operatorname{dcl}_{\mathrm{T}}\left(\operatorname{acl}_{\mathrm{T}}(K), \operatorname{Fix}(M, \sigma)\right)=\operatorname{acl}_{\mathrm{T}}(\operatorname{Fix}(M, \sigma)),
$$


then any $\alpha \in \operatorname{Aut}_{L}(\operatorname{Fix}(M, \sigma \phi) / K)$ lifts to an automorphism of $(M, \sigma)$ over $K$. Namely, $(M, \sigma \phi)$ is a model of $T A$ by remark (2.1.1), so by the lemma $\alpha$ lifts to an automorphism $\tilde{\alpha}$ of $(M, \sigma \phi)$ over $K$, which is an automorphism of the $L_{\sigma}$-structure $(M, \sigma)$ by remark (2.1.1).

Proof of lemma (3.6). Note first that any automorphism $\alpha$ of $(M, \sigma)$ restricts to an automorphism of the $L$-structure $\operatorname{Fix}(M, \sigma)$ because the latter is definable in $(M, \sigma)$ without parameters.

Now let $\alpha \in \operatorname{Aut}_{L}(\operatorname{Fix}(M, \sigma) / K) . \alpha$ is a partial elementary map in the sense of $T$, see remark (2.2), and commutes with $\left.\sigma\right|_{\operatorname{Fix}(M, \sigma)}$ simply because $\sigma$ is the identity on $\operatorname{Fix}(M, \sigma)$. We are going to lift $\alpha$ to a permutation $\tilde{\alpha}$ of $\operatorname{acl}_{\mathrm{T}}(F)$ that is elementary in the sense of $T$ and commutes with $\sigma \cdot \operatorname{As~acl}_{\mathrm{T}}(\operatorname{Fix}(M, \sigma))=$ $\operatorname{acl}_{\sigma}(\operatorname{Fix}(M, \sigma))$ by fact (2.4.2), fact (2.4.1) then implies that $\tilde{\alpha}$ is elementary in the sense of $T A$. Then in particular $\alpha$ is an automorphism of the induced structure from $(M, \sigma)$ on $\operatorname{Fix}(M, \sigma)$. So by saturation of $(M, \sigma)$, lemma $(2.7)$ implies that $\alpha$ lifts to an automorphism of $(M, \sigma)$ over $K$.

To lift $\alpha$ we work entirely in the $L$-structure $M$. In particular, types, independence and so on are all ment to be in the sense of $T$, not $T A$.

Let $\bar{a} \in \operatorname{Fix}(M, \sigma)$ and $\bar{b} \in \operatorname{acl}_{\mathrm{T}}(K)$. As $\operatorname{acl}_{\mathrm{T}}(K) \cap \operatorname{Fix}(M, \sigma)=K$ by lemma (3.4) and because $\alpha$ leaves $K$ pointwise fixed, it follows from lemma (3.5) that $\operatorname{tp}_{T}(\bar{a} / K)$ is stationary. So $\operatorname{tp}_{T}(\alpha(\bar{a}) / K)$ is stationary, too, and equals $\operatorname{tp}_{T}(\bar{a} / K)$. As $\bar{b}$ is independent from $\operatorname{Fix}(M, \sigma)$ over $K$ it follows that $\operatorname{tp}_{T}(\bar{a} / K \bar{b})$ equals $\operatorname{tp}_{T}(\alpha(\bar{a}) / K \bar{b})$, which in turn implies that $\operatorname{tp}_{T}(\bar{a}, \bar{b})=\operatorname{tp}_{T}(\alpha(\bar{a}), \bar{b})$. So the map id $\left.\right|_{\operatorname{acl}_{T}(K)} \cup \alpha$ is elementary in the sense of $T$. It lifts uniquely to an elementary permutation $\tilde{\alpha}$ of $\operatorname{dcl}_{\mathrm{T}}\left(\operatorname{acl}_{\mathrm{T}}(K), \operatorname{Fix}(M, \sigma)\right)$, which is $\operatorname{acl}_{\mathrm{T}}(\operatorname{Fix}(M, \sigma))$ by assumption. $\tilde{\alpha}$ commutes with $\sigma$ by construction, so we are done.

Proposition 3.7 Let $T$ be a stable L-theory with quantifier elimination and elimination of imaginaries and let $\phi$ be an automorphism of $T$ which is $L$ definable over $\emptyset$. Assume that $T A$ exists and has elimination of imaginaries.

Let $(M, \sigma)$ be a model of $T A$ and $K \preccurlyeq_{L} \operatorname{Fix}(M, \sigma \phi)$. If

$$
\operatorname{dcl}_{\mathrm{T}}\left(\operatorname{acl}_{\mathrm{T}}(K), \operatorname{Fix}(M, \sigma \phi)\right)=\operatorname{acl}_{\mathrm{T}}(\operatorname{Fix}(M, \sigma \phi)),
$$

then $\operatorname{Fix}(M, \sigma \phi)$ is conservatively embedded over $K$ in $(M, \sigma)$.

Proof. First note that if $(N, \sigma)$ is an elementary extension of $(M, \sigma)$, then $\operatorname{Fix}(M, \sigma \phi)$ is conservatively embedded over $K$ in $(M, \sigma)$ if and only if $\operatorname{Fix}(N, \sigma \phi)$ is so in $(N, \sigma)$ by lemma (3.2). Furthermore it follows from proposition (3.3) that $\operatorname{dcl}_{\mathrm{T}}\left(\operatorname{acl}_{\mathrm{T}}(K), \operatorname{Fix}(N, \sigma \phi)\right)=\operatorname{acl}_{\mathrm{T}}(\operatorname{Fix}(N, \sigma \phi))$. So we may assume that $(M, \sigma)$ is sufficiently saturated.

Let $X$ be a subset of (some cartesian power of) $\operatorname{Fix}(M, \sigma \phi)$ that is $K$-definable in $(M, \sigma)$. As we have noted above, it follows from lemma (3.6) that we can lift any automorphism of $\operatorname{Fix}(M, \sigma \phi)$ over $K$ to an automorphism of $(M, \sigma \phi)$ over $K$, which is an automorphism of $(M, \sigma)$ over $K$ as well by remark (2.1.1). This shows that for any tuple $a \in \operatorname{Fix}(M, \sigma \phi)$ the type of $a$ over $K$ in the sense of $\operatorname{Fix}(M, \sigma \phi)$ (relativised to $\sigma \phi(x)=x)$ implies modulo the theory of $(M, \sigma)$ the type of $a$ over $K$ in $(M, \sigma)$. Hence by compactness $X$ is definable in the $L$-structure $\operatorname{Fix}(M, \sigma \phi)$ using parameters from $K$. 
Taking $K=\operatorname{Fix}(M, \sigma \phi)$ we obtain the following result as a special case of proposition (3.7). It was proved before in the cases of fields: when $T$ is $A C F$ by Chatzidakis, Hrushovski and Peterzil in [5], for separably closed fields with generic automorphism by Chatzidakis in [3], when $T$ is $D C F_{0}$ by BustamanteMedina in [2] and for strongly minimal $T$ by Pillay in [12].

Corollary 3.8 Let $T$ be a stable L-theory with quantifier elimination and elimination of imaginaries and let $\phi$ be an automorphism of $T$ which is L-definable over $\emptyset$. Assume that $T A$ exists and eliminates imaginaries. Let $(M, \sigma)$ be a model of $T A$. Then every subset $X$ of (some cartesian power of) $\operatorname{Fix}(M, \sigma \phi)$ definable in $(M, \sigma)$ using parameters is definable in the L-structure $\operatorname{Fix}(M, \sigma \phi)$ using parameters.

\section{Prescribed Fixed Structures}

In this section we prove our main theorem and discuss some variants.

Theorem 4.1 Let $T$ be a countable complete stable L-theory with quantifier elimination and elimination of imaginaries. Assume that $T A$ exists and has elimination of imaginaries. Let $(M, \sigma)$ be a model of $T A$ and $K \preccurlyeq_{L} \operatorname{Fix}(M, \sigma)$. Then the following are equivalent:

(1) There is some $(N, \sigma) \equiv(M, \sigma)$ with $\operatorname{Fix}(N, \sigma)=K$.

(2) $\operatorname{Fix}(M, \sigma)$ is conservatively embedded over $K$ in $(M, \sigma)$.

(3) $\operatorname{dcl}_{\mathrm{T}}\left(\operatorname{acl}_{\mathrm{T}}(K), \operatorname{Fix}(M, \sigma)\right)=\operatorname{acl}(\operatorname{Fix}(M, \sigma))$.

(4) $\operatorname{Gal}(K)=\widehat{\mathbb{Z}}$.

Needless to say that as a corollary we obtain theorem (1.1).

Proof. That (1) implies (4) is remark (2.3) because of elimination of imaginaries.

To see that (4) implies (3), note that $K=\operatorname{acl}_{\mathrm{T}}(K) \cap \operatorname{Fix}(M, \sigma)$ by lemma (3.4), so by Galois theory ([14]) we have $K=\operatorname{Fix}\left(\operatorname{acl}_{\mathrm{T}}(K),\left.\sigma\right|_{\operatorname{acl}_{\mathrm{T}}(K)}\right)$. Hence the natural restriction map res : $\operatorname{Gal}(\operatorname{Fix}(M, \sigma)) \longrightarrow \operatorname{Gal}(K)$ is surjective. As both Galois groups equal $\widehat{\mathbb{Z}}$, res must be an isomorphism because any surjective endomorphism of bounded ${ }^{1}$ profinite groups is an automorphism. So the assertion follows as in the proof of proposition (3.3).

That (3) implies (2) is the content of proposition (3.7).

We finally prove that (2) implies (1). In view of lemma (3.2) we may assume that $(M, \sigma)$ is sufficiently ${ }^{2}$ saturated. We construct $(N, \sigma)$ with the aid of the following standard chain argument. Starting with $N_{0}=\operatorname{acl}_{\sigma}(K)$, whose set of elements fixed by $\sigma$ is precisely $K$ by (3.4), we build an ascending chain $\left(N_{\nu}\right)_{\nu<\omega}$ of acl $\sigma_{\sigma}$-closed subsets of $(M, \sigma)$ with the property that every consistent quantifier-free $L_{\sigma}\left(N_{\nu}\right)$-formula has a realisation in $N_{\nu+1}$ and furthermore that

\footnotetext{
${ }^{1}$ recall that a profinite group $G$ is called bounded if for any natural number $n$ there are up to isomorphism only finitely many homomorphic images of order $n$

2 in fact we only need that $(M, \sigma)$ is $|K|^{+}$-saturated.
} 
$\operatorname{Fix}(M, \sigma) \cap N_{\nu}=K$ for all $\nu<\omega$. Then we let $N=\bigcup N_{\nu}$. By model completeness of $T A$, any $L_{\sigma}$-formula is equivalent modulo $T A$ to an existential formula. So by Tarski's Test $\left(N,\left.\sigma\right|_{N}\right)$ will be an elementary substructure of $(M, \sigma)$, with $\operatorname{Fix}(N, \sigma)=K$ by construction.

The only delicate point is to ensure $\operatorname{Fix}\left(N_{\nu}, \sigma\right)=K$ for all $\nu<\omega$. So let $A$ be an $\operatorname{acl}_{\sigma}$-closed subset of $M$ such that $\operatorname{Fix}(A, \sigma)=K$ and $\varphi(\bar{x})$ be a consistent quantifier-free $L_{\sigma}(A)$-formula. $T A$ is quantifier-free stable by lemma (2.5) and countable because $T$ is. By proposition (2.6) and by saturation of $(M, \sigma)$ we can choose a tuple $\bar{a} \in M$ satisfying $\varphi$ whose quantifier-free type $\mathrm{qftp}_{\sigma}(\bar{a} / A)$ over $A$ in the sense of $(M, \sigma)$ is locally isolated. Then the following proposition (4.2) implies that $\operatorname{acl}_{\sigma}(A, \bar{a}) \cap \operatorname{Fix}(M, \sigma \phi)=\operatorname{Fix}(A, \sigma)$.

Proposition 4.2 Let $T$ and $T A$ be as in theorem (4.1) and $(M, \sigma) \models T A$. Let $A$ be an $\operatorname{acl}_{\sigma}$-closed subset of $(M, \sigma)$ such that $\operatorname{Fix}(A, \sigma) \preccurlyeq_{L} \operatorname{Fix}(M, \sigma)$. If $\operatorname{Fix}(M, \sigma)$ is conservatively embedded over $\operatorname{Fix}(A, \sigma)$ in $(M, \sigma)$, then

$$
\operatorname{acl}_{\sigma}(A, \bar{a}) \cap \operatorname{Fix}(M, \sigma)=\operatorname{Fix}(A, \sigma)
$$

for any tuple $\bar{a} \in M$ whose quantifier-free $L_{\sigma}$-type $\operatorname{qftp}_{\sigma}(\bar{a} / A)$ over $A$ is locally isolated.

Proof. Let $\bar{a}$ be a tuple in $M$ whose quantifier-free $L_{\sigma}$-type over $A$ is locally isolated. We abbreviate $\operatorname{Fix}(A, \sigma)$ by $K$ and show first that

$$
\operatorname{dcl}_{\mathrm{T}}\left(\operatorname{cl}_{\sigma}(A, \bar{a})\right) \cap \operatorname{Fix}(M, \sigma)=K .
$$

Let $b \in \operatorname{dcl}_{\mathrm{T}}\left(\operatorname{cl}_{\sigma}(A, \bar{a})\right)$ be fixed by $\sigma$. Then $b$ is $L$-definable over

$$
A, \sigma^{-n}(\bar{a}), \ldots, \sigma^{-1}(\bar{a}), \bar{a}, \sigma(\bar{a}), \ldots, \sigma^{n}(\bar{a})
$$

for some $n \in \mathbb{N}$, and, as $\sigma(b)=b$, applying $\sigma^{n}$ we see that $b$ is already $L$ definable over $A, \bar{a}, \ldots, \sigma^{n}(\bar{a})$ for some $n \in \mathbb{N}$ since $\sigma$ is an $L_{\sigma}$-automorphism. So there is some $L$-formula

$$
\psi\left(\bar{x}_{0}, \bar{x}_{1}, \ldots, \bar{x}_{n} ; z, \bar{y}\right)
$$

and $\bar{e} \in A$ such that $\psi\left(\bar{a}, \sigma(\bar{a}), \ldots, \sigma^{n}(\bar{a}) ; z, \bar{e}\right)$ defines $b$ in $M$. As $T$ eliminates quantifiers, we may assume that $\psi$ is quantifier-free. Let

$$
\Delta=\left\{\psi\left(\bar{x}, \sigma(\bar{x}), \ldots, \sigma^{n}(\bar{x}) ; z, \bar{y}\right), \sigma(z)=z\right\}
$$

and choose an $L_{\sigma}(A)$-formula $\delta(\bar{x}) \in \operatorname{qftp}_{\sigma}(\bar{a} / A)$ isolating $\left.\operatorname{qftp}_{\sigma}(\bar{a} / A)\right|_{\Delta}$.

Consider the $L_{\sigma}$-formula

$$
\Phi(z)=\exists \bar{x} \quad\left(\delta(\bar{x}) \wedge \psi\left(\bar{x}, \sigma(\bar{x}), \ldots, \sigma^{n}(\bar{x}) ; z, \bar{e}\right) \wedge \sigma(z)=z\right)
$$

with parameters from $A$ and let $X$ be the subset of $\operatorname{Fix}(M, \sigma)$ defined by $\Phi$.

We claim that $X$ is $L_{\sigma}$-definable in $(M, \sigma)$ over $A \cap \operatorname{Fix}(M, \sigma)$. To see this, note that both $A$ and $\operatorname{Fix}(M, \sigma)$ are $\mathrm{dcl}_{\sigma}$-closed. $T A$ eliminates imaginaries by assumption, so on the one hand $\operatorname{Fix}(M, \sigma)$ is stably embedded by remark (2.8), whence the canonical parameter of $X$ is in $\operatorname{Fix}(M, \sigma)$. On the other hand the 
canonical parameter of $X$ is also in $A$. Thus $X$ is $L_{\sigma}$-definable in $(M, \sigma)$ over $A \cap \operatorname{Fix}(M, \sigma)=K$.

By assumption $\operatorname{Fix}(M, \sigma)$ is conservatively embedded over $K$ in $(M, \sigma)$, so it follows that $X$ is $L$-definable over $K$ in the $L$-structure $\operatorname{Fix}(M, \sigma) . X$ is nonempty because $b \in X$, hence $\operatorname{Fix}(M, \sigma) \models \exists z z \in X$. But $K \preccurlyeq_{L} \operatorname{Fix}(M, \sigma)$, so $K \models \exists z z \in X$, whence there is $\lambda \in X(K)$.

We have shown that the $L_{\sigma}(A)$-formula

$$
\psi\left(\bar{x}, \sigma(\bar{x}), \ldots, \sigma^{n}(\bar{x}) ; \lambda, \bar{e}\right)
$$

is consistent with $\delta(\bar{x})$ for some $\lambda \in K$. Thus

$$
\psi\left(\bar{x}, \sigma(\bar{x}), \ldots, \sigma^{n}(\bar{x}) ; \lambda, \bar{e}\right) \in \operatorname{qftp}_{\sigma}(\bar{a} / A)
$$

and therefore $b=\lambda \in K$, because $\psi\left(\bar{a}, \ldots, \sigma^{n}(\bar{a}) ; z, \bar{e}\right)$ has exactly one solution in $M$. This shows that $\operatorname{dcl}_{\mathrm{T}}\left(\operatorname{cl}_{\sigma}(A, \bar{a})\right) \cap \operatorname{Fix}(M, \sigma)=K$.

Finally we show that any tuple $\bar{a}$ with $\operatorname{dcl}_{\mathrm{T}}\left(\operatorname{cl}_{\sigma}(A, \bar{a})\right) \cap \operatorname{Fix}(M, \sigma)=K$ has the property that

$$
\operatorname{acl}_{\sigma}(A, \bar{a}) \cap \operatorname{Fix}(M, \sigma)=K .
$$

Indeed, by fact (2.4.2) we have $\operatorname{acl}_{\sigma}(A, \bar{a})=\operatorname{acl}_{\mathrm{T}}\left(\operatorname{dcl}_{\mathrm{T}}\left(\mathrm{cl}_{\sigma}(A, \bar{a})\right)\right)$, so the next lemma (4.3) implies that any $b \in \operatorname{acl}_{\sigma}(A, \bar{a})$ which is fixed by $\sigma$ is in the $\operatorname{acl}_{\mathrm{T}^{-}}$ closure of

$$
\operatorname{dcl}_{\mathrm{T}}\left(\operatorname{cl}_{\sigma}(A, \bar{a})\right) \cap \operatorname{Fix}(M, \sigma)=K .
$$

It follows that $b \in \operatorname{acl}_{\mathrm{T}}(K)$. As $A$ is algebraically closed and contains $K$ we conclude that $b \in A$ and thus, as $\sigma(b)=b$, that $b \in K$.

Lemma 4.3 Let $T^{\prime}$ be any theory with elimination of imaginaries and $B$ a definably-closed set. If $\alpha$ is an automorphism of a sufficiently saturated model mapping $B$ into itself and if $b$ algebraic over $B$ and fixed by $\alpha$, then $b$ is algebraic over $B \cap \operatorname{Fix}(\alpha)$.

Proof. Choose an $L(B)$-formula $\psi$ isolating the type of $b$ over $B$. As $b$ is fixed by $\alpha, \psi$ is invariant under $\alpha$ and thus the canonical parameter for $\psi$ is in $B \cap \operatorname{Fix}(\alpha)$.

The proof of theorem (4.1) is complete.

For the applications in the next section, the following corollary will be suitable.

Corollary 4.4 Let T,TA be as in theorem (4.1) and $(M, \sigma)$ be a model of TA. Let further $A$ be an acl $_{\sigma}$-closed subset of $M$ and denote $K=\operatorname{Fix}(A, \sigma)$. If $\operatorname{Gal}(K)=\widehat{\mathbb{Z}}$ and $K \preccurlyeq_{L} \operatorname{Fix}(M, \sigma)$, then $(A, \sigma)$ embeds into a model $(N, \sigma)$ of $T A$ such that $\operatorname{Fix}(N, \sigma)=K$.

We now discuss two variants of theorem (4.1). The first is a strengthening in case $T$ is totally transcendental. Let us examine once more the proof of theorem (4.1). The only place where we use saturation of $(M, \sigma)$ is where we realise a locally isolated quantifier-free type containing the formula $\varphi(\bar{x})$. As mentioned earlier in lemma (2.5), $T A$ is quantifier-free totally transcendental if $T$ is totally 
transcendental. So in this case proposition (2.6) allows us to choose a tuple $\bar{a}$ realising $\varphi(\bar{x})$ whose quantifier-free type is even isolated, rather than locally isolated only. For such $\bar{a}$, following the proof of (4.2) word by word, we then obtain $\operatorname{acl}_{\sigma}(A \bar{a}) \cap \operatorname{Fix}(M, \sigma)=K$. So we have proved the following theorem.

Theorem 4.5 Let $T$ be a complete totally transcendental L-theory with quantifier elimination and elimination of imaginaries. Assume that $T A$ exists and has elimination of imaginaries.

Let $(M, \sigma)$ be a model of $T A$ and $K \preccurlyeq_{L} \operatorname{Fix}(M, \sigma)$. Then the following are equivalent:

(0) There is some $(N, \sigma) \equiv(M, \sigma)$ with $\operatorname{Fix}(N, \sigma)=K$.

(1) There is some $(N, \sigma) \preccurlyeq(M, \sigma)$ with $\operatorname{Fix}(N, \sigma)=K$.

(2) $\operatorname{Fix}(M, \sigma)$ is conservatively embedded over $K$ in $(M, \sigma)$.

(3) $\operatorname{dcl}_{\mathrm{T}}\left(\operatorname{acl}_{\mathrm{T}}(K), \operatorname{Fix}(M, \sigma)\right)=\operatorname{acl}_{\mathrm{T}}(\operatorname{Fix}(M, \sigma))$.

(4) $\operatorname{Gal}(K)=\widehat{\mathbb{Z}}$.

For the second variant of theorem (4.1), let $T$ and $T A$ be as in that theorem. Let $(M, \sigma)$ be a model of $T A$ and $A=\operatorname{acl}_{\sigma}(A)$ be a subset of $M$. Consider an automorphism $\phi$ of $T$ which is $L$-definable over $\emptyset$. Then by remark $(2.1 .1),(M, \sigma \phi)$ is a model of $T A$, too, and $A$ is acl $\sigma^{\text {-closed in }}(M, \sigma \phi)$. Furthermore, by the same remark, the quantifier-free type in the sense of $(M, \sigma)$ of a tuple $\bar{a} \in M$ is locally isolated in $(M, \sigma)$ if and only if the quantifier-free type in the sense of $(M, \sigma \phi)$ of $\bar{a}$ is locally isolated in $(M, \sigma \phi)$. Hence if $K=\operatorname{Fix}(A, \sigma \phi) \preccurlyeq L \operatorname{Fix}(M, \sigma \phi)$ and $\operatorname{dcl}_{\mathrm{T}}\left(\operatorname{acl}_{\mathrm{T}}(K), \operatorname{Fix}(M, \sigma \phi)\right)=\operatorname{acl}_{\mathrm{T}}(\operatorname{Fix}(M, \sigma \phi))$, then $\operatorname{Fix}(M, \sigma \phi)$ is conservatively embedded over $K$ in $(M, \sigma \phi)$ by proposition (3.7), and so by proposition (4.2) we get

$$
\operatorname{acl}_{\sigma}(A, \bar{a}) \cap \operatorname{Fix}(M, \sigma \phi)=\operatorname{Fix}(A, \sigma \phi) .
$$

The same argument applies if we consider several $\phi$ 's at once, so the proof of theorem (4.1) shows also the following corollary, which in the case when $T$ is $A C F$ was proved independently by Zoé Chatzidakis (unpublished).

Corollary 4.6 Let $T$ and $T A$ be as in theorem (4.1). Let further $\left(\phi_{i}\right)_{i \in I}$ be a family of $L$-automorphisms of $T$, with each $\phi_{i} L$-definable over $\emptyset$, and $(M, \sigma)$ be a model of $T A$. If $A$ is an $\mathrm{acl}_{\sigma}$-closed subset of $(M, \sigma)$ such that for all $i \in I$, $\operatorname{Fix}\left(A, \sigma \phi_{i}\right) \preccurlyeq_{L} \operatorname{Fix}\left(M, \sigma \phi_{i}\right)$ and

$$
\operatorname{dcl}_{\mathrm{T}}\left(\operatorname{acl}_{\mathrm{T}}\left(\operatorname{Fix}\left(A, \sigma \phi_{i}\right)\right), \operatorname{Fix}\left(M, \sigma \phi_{i}\right)\right)=\operatorname{acl}_{\mathrm{T}}\left(\operatorname{Fix}\left(M, \sigma \phi_{i}\right)\right),
$$

then there is a model $(N, \sigma) \equiv(M, \sigma)$ of $T A$ such that for all $i \in I$

$$
\operatorname{Fix}\left(N, \sigma \phi_{i}\right)=\operatorname{Fix}\left(A, \sigma \phi_{i}\right) .
$$

As before, if $T$ is totally transcendental, we can choose $(N, \sigma)$ to be an elementary submodel of $(M, \sigma)$. 


\section{$5 \quad$ Fixed Fields of Generic Automorphisms}

We now apply the results of the previous section to the case of generic automorphisms of fields. For a field $k$ we denote $\operatorname{abs}(k)$ the absolute part of $k$, which is the subfield of elements algebraic over the prime field. Frob denotes the Frobenius homomorphism in positive characteristic. Recall that a field is called one-free if its absolute Galois group is $\widehat{\mathbb{Z}}$. Recall also that a difference field $(F, \sigma)$ is a field $F$ endowed with an automorphism $\sigma$ and a difference-differential field $(F, d, \sigma)$ is a differential field $(F, d)$ endowed with a field automorphism $\sigma$ that commutes with the derivation $d$. We call $\operatorname{Fix}(F, d, \sigma)$ the fixed differential field of $(F, d, \sigma)$, which is the subdifferential field of $(F, d)$ whose elements are fixed by $\sigma$. Our reference for algebra is [10], and [7] for difference fields. For the model theory of difference fields we refer to [4], and for difference-differential fields to [2]. We give further references below.

We will need the following algebraic lemma.

Lemma 5.1 Any difference-differential field $(F, d, \sigma)$ whose fixed differential field $(k, d)$ has procyclic absolute Galois group admits an extension $\left(F^{\mathrm{sep}}, \bar{d}, \bar{\sigma}\right)$ with $\operatorname{Fix}\left(F^{\mathrm{sep}}, \bar{d}, \bar{\sigma}\right)=(k, d)$.

Proof. Recall from [11] that the derivation $d$ on $F$ extends uniquely to a derivation $\bar{d}$ on $F^{\text {sep }}$. If $\alpha \in F^{\text {sep }}$ and $p(X) \in F[X]$ is the normalised minimal polynomial of $\alpha$ over $F$, then $\bar{d}(\alpha)=-\frac{p^{d}(\alpha)}{p^{\prime}(\alpha)}$, where $p^{\prime}$ is the formal derivative of $p$ and $p^{d}$ is the polynomial obtained by applying $d$ to the coefficients of $p$, so one computes right away that any extension of $\sigma$ to $F^{\text {sep }}$ commutes with $\bar{d}$.

Thus we only have to show that there is some extension $\bar{\sigma}$ of $\sigma$ to $F^{\text {sep }}$ such that $\operatorname{Fix}\left(F^{\mathrm{sep}}, \bar{\sigma}\right)=\operatorname{Fix}(F, \sigma)$. Let to that end $L=F \cap k^{\text {sep }}$ be the elements of $F$ separably algebraic over $k$. Since $k$ has procyclic absolute Galois group, any element $x \in L$, which generates the unique (in $k^{\text {sep }}$ ) algebraic extension of $k$ of degree $[k[x]: k]$, is mapped into $L$ by all automorphisms of $k^{\text {sep }}$ over $k$. Therefore $L$ is invariant under $\operatorname{Gal}\left(k^{\mathrm{sep}} / k\right)$. As $\sigma$ transforms algebraic elements into algebraic ones, it restricts to an automorphism of $L$ with fixed field $k$. Thus $L / k$ is Galois and $\left.\sigma\right|_{L}$ generates $\operatorname{Gal}(L / k)$.

Now $\left.\sigma\right|_{L}$ extends to a topological generator $\tau$ of $\operatorname{Gal}\left(k^{\mathrm{sep}} / k\right)$ because $\operatorname{Gal}\left(k^{\mathrm{sep}} / k\right)$ is procyclic and res : $\operatorname{Gal}\left(k^{\mathrm{sep}} / k\right) \rightarrow \operatorname{Gal}(L / k)$ is surjective. Of course $k^{\mathrm{sep}} / L$ is a Galois extension and thus by choice of $L, F$ and $k^{\text {sep }}$ are linearly disjoint over $L$. So as $\sigma$ and $\tau$ agree on $L$ we can find a (unique) automorphism of $F k^{\text {sep }}$ extending $\sigma$ and $\tau$. We lift it to an automorphism $\bar{\sigma} \in \operatorname{Aut}\left(F^{\text {sep }}\right)$ and claim that $\operatorname{Fix}\left(F^{\mathrm{sep}}, \bar{\sigma}\right)=\operatorname{Fix}(F, \sigma)=k$. To see this, note first that $\operatorname{Fix}\left(F^{\mathrm{sep}}, \bar{\sigma}\right) \subset \operatorname{Fix}(F, \sigma)^{\mathrm{sep}}$. Indeed, let $p \in F[X]$ be the minimal normalised polynomial of some $x \in \operatorname{Fix}\left(F^{\mathrm{sep}}, \bar{\sigma}\right)$. Because $\bar{\sigma}(x)=x$, one calculates that $p^{\sigma}(x)=p^{\sigma}(\bar{\sigma}(x))=\bar{\sigma}(p(x))=0$, where $p^{\sigma}$ denotes the polynomial obtained by applying $\sigma$ to the coefficients of $p$. This implies $p=p^{\sigma}$ and thus that all coefficients of $p$ are contained in $\operatorname{Fix}(F, \sigma)$, so $x \in k^{\text {sep }}$. Finally, as $\tau$ is a topological generator of $\operatorname{Gal}\left(k^{\mathrm{sep}} / k\right)$, it follows that $x \in k$. 


\subsection{Pseudofinite fields and models of $A C F A$}

Let $L$ be the language of rings and $A C F$ be the theory of algebraically closed fields (of a fixed characteristic), which is $\omega$-stable and has quantifier elimination and elimination of imaginarires. $A C F A$ exists and eliminates imaginaries. Given any pseudofinite field $k$, there is some model of $A C F A$ whose fixed field is elementarily equivalent to $k$. For more details and proofs about $A C F A$ we refer to [4], and to [1] for pseudofinite fields.

Theorem 5.2 Any difference field whose fixed field $k$ is pseudofinite embeds into some model of $A C F A$ with fixed field $k$.

Proof. Obviously any difference field can be considered a difference-differetial field by adding the trivial derivation. So by lemma (5.1) we may assume that our difference field, call it $(F, \sigma)$, is separably closed. Further we may assume that $(F, \sigma)$ is algebraically closed, because $\sigma$ lifts uniquely to an automorphism $\bar{\sigma}$ of $F^{\text {alg }}$, and one has $\operatorname{Fix}\left(F^{\text {alg }}, \bar{\sigma}\right)=\operatorname{Fix}(F, \sigma)^{\text {perf }}=\operatorname{Fix}(F, \sigma)$.

$(F, \sigma)$ embeds into some model $(\Omega, \sigma)$ of $A C F A$, and is $\operatorname{acl}_{\sigma}$-closed therein. $\operatorname{abs}(k)=\operatorname{abs}(\operatorname{Fix}(\Omega, \sigma))$ and thus, both fields being pseudofinite, $k \preccurlyeq \operatorname{Fix}(\Omega, \sigma)$. Hence corollary (4.4) finishes the proof.

Corollary 5.3 Any pseudofinite field $k$ is isomorphic to the fixed field of some model of $A C F A$. In positive characteristic, if $\operatorname{abs}(k)=\operatorname{Fix}\left(\mathbb{F}_{p}^{\text {alg }}, \sigma \circ\right.$ Frob $\left.^{n}\right)$ for some $\sigma \in \operatorname{Gal}\left(\mathbb{F}_{p}\right)$ and $n \in \mathbb{Z}$, then there is some model $(K, \sigma)$ of $A C F A$ such that $\operatorname{Fix}\left(K, \sigma \circ \operatorname{Frob}^{n}\right)=k$.

Proof. To show the first assertion, apply theorem (5.2) to the difference field $(k, \mathrm{id})$. For the second let $\operatorname{char}(k)=p>0$. Again because $\operatorname{Gal}(\operatorname{abs}(k))$ is procyclic and the natural restriction map res : $\operatorname{Gal}(k) \longrightarrow \operatorname{Gal}(\operatorname{abs}(k))$ is surjective, $\sigma$ lifts to an automorphism $\bar{\sigma}$ of $k^{\text {alg }}$ such that $\bar{\sigma} \circ$ Frob $^{n}$ is a topological generator of $\operatorname{Gal}(k)$. By theorem (5.2) the difference field $\left(k^{\text {alg }}, \sigma \circ\right.$ Frob $\left.^{n}\right)$ embeds into some model $(\Omega, \tau)$ of $A C F A$ with $\operatorname{Fix}(\Omega, \tau)=k$, and setting $\sigma=\tau \circ \mathrm{Frob}^{-n}$, we obtain a model $(\Omega, \sigma)$ of $A C F A$ with $\operatorname{Fix}\left(\Omega, \sigma \circ \operatorname{Frob}^{n}\right)=k$.

Note that any two different choices of topological generators of $\mathrm{Gal}(k)$ give nonelementarily equivalent models of $A C F A$ with fixed field $k$ by applying theorem (5.2) to the difference field $\left(k^{\text {alg }}, \sigma\right)$.

As an application of corollary (4.6) we obtain in positive characteristic the following theorem, which was pointed out to us and proved independently by Zoé Chatzidakis (unpublished).

Theorem 5.4 (Chatzidakis) Let $(K, \sigma)$ be an algebraically closed difference field and $\Sigma \subseteq \mathbb{Z}$ such that for all $n \in \Sigma$ the fixed fields $\operatorname{Fix}\left(K, \sigma \circ \operatorname{Frob}^{n}\right)$ are pseudofinite. Then there is some model $(\Omega, \sigma)$ of $A C F A$ such that for all $n \in \Sigma$

$$
\operatorname{Fix}\left(\Omega, \sigma \circ \operatorname{Frob}^{n}\right)=\operatorname{Fix}\left(K, \sigma \circ \operatorname{Frob}^{n}\right) .
$$




\subsection{One-free pseudo-differentially closed fields and models of $D C F A$}

Let $L$ be the language of differential fields, which is the ring language together with a unary function symbol $d$, and let $T$ be the theory $D C F_{0}$ of differentially closed fields. $D C F_{0}$ is $\omega$-stable and has elimination of quantifiers and of imaginaries. Hrushovski shows (in unpublished work) that DCFA exists, and elimination of imaginaries for $D C F A$ was proved by Bustamante-Medina in [2]. There it is also shown that if $(k, d)$ is a differential field, then $(k, d) \equiv \operatorname{Fix}(\Omega, d, \sigma)$ for some model $\operatorname{Fix}(\Omega, d, \sigma)$ of $D C F A$ if and only if

1. $k$ is pseudofinite of characteristic zero, and

2. $(k, d)$ satisfies the geometric axioms of differentially closed fields. Namely for every (absolutely irreducible) variety $V$ over $k$ and (abs. irred.) subvariety $W$ of the torsor $\tau(V)$ of $V$ projecting generically onto $V$, there is $a \in V(k)$ such that $(a, d(a)) \in W$.

We refer to [2] for more details and proofs. We call a differential field of characteristic zero pseudo-differentially closed if it satisfies 2 . Note that $(k, d)$ is one-free pseudo-differentially closed if and only if it is elementarily equivalent to some fixed differential field of some model of $D C F A$.

Theorem 5.5 An difference-differential field whose fixed differential field $(k, d)$ is one-free pseudo-differentially closed embeds into a model $(\Omega, d, \sigma)$ of DCF A such that $\operatorname{Fix}(\Omega, d, \sigma)=(k, d)$.

Proof. By lemma (5.1) and because the characteristic is zero, we may assume that our difference-differential field, call it $(F, d, \sigma)$, is algebraically closed. We embed it into some model of $(\Omega, d, \sigma)$ of $D C F A$. Then $k$ and $\operatorname{Fix}(\Omega, d, \sigma)$ are elementarily equivalent as pure fields, and hence by proposition (5.8) of [13]), the differential fields $(k, d)$ and $\operatorname{Fix}(\Omega, d, \sigma)$ are elementarily equivalent because both are pseudo-differentially closed. As $F$ is algebraically closed in the sense of $D C F_{0}$ and $\sigma$ is an automorphism of $(F, d)$, we see that $F$ is acl $\sigma^{\text {-closed. }}$ As furthermore $k$ alg is the algebraic closure of $k$ in the sense of $D C F_{0}$ and any field automorphism of $k^{\text {alg }}$ commutes with the derivation on $k^{\text {alg }}$, we have $\operatorname{Gal}(k)=\widehat{\mathbb{Z}}$. Hence corollary (4.4) finishes the proof.

Corollary 5.6 Any one-free pseudo-differentially closed field is isomorphic to the fixed differential field of some model of DCFA.

Proof. Apply theorem (5.5) to the difference-differential field $(k, d$, id).

Note that, as above, any choice of a topological generator of the absolute Galois group of $k$ yields non-elementarily equivalent models of $D C F A$ having fixed differential field $(k, d)$.

\subsection{One-free PAC fields and models of $S C F A$}

We fix a prime number $p$ and a positive natural number $e$. Let $L$ be the ring language augmented by $e$ constant symbols $b_{1}, \ldots, b_{e}$ for $p$-bases, and let $S C F_{e, b}$ 
be the $L$-theory of separably closed fields of Ershov invarant $e . S C F_{e, b}$ is model complete, stable and has elimination of imaginaries. Our reference for separably closed fields is [8]. Chatzidakis shows in [3] that the model companion of $\left(S C F_{e, b}\right)_{\sigma}$ exists, which we denote by $S C F A_{e}$, and that $S C F A_{e}$ has elimination of imainaries. (Our $S C F A_{e}$ is the $S C F A_{e, b}$ of [3].) It is clear from the description of the completions of $S C F A_{e}$ given in [3] that for every one-free PAC field $k$ of degree of imperfection $e$ there is some model of $S C F A_{e}$ whose fixed field is elementarily equivalent to $k$. For details and proofs about one-free $P A C$ fields we refer to [9].

Theorem 5.7 Any difference field whose fixed field $k$ is one-free $P A C$ and of finite Ershov invariant e embeds into some model $(\Omega, \sigma)$ of $S C F A_{e, b}$ such that $\operatorname{Fix}(\Omega, \sigma)=k$.

Before we give the proof, let us make the following simple observations. If $(F, \sigma)$ is any difference field, and if $\bar{c}$ is a tuple fixed by $\sigma$ such that there is some $p$-monomial in $\bar{c}$ which can be expressed as a minimal non-trivial linear combination of other $p$-monomials in $\bar{c}$ with coefficients in $F^{p}$, then these coefficients must also be fixed by $\sigma$. So any set of $p$-independent elements in $\operatorname{Fix}(F, \sigma)$ is $p$-independent in $F$, or equivalently the field extension $F / \operatorname{Fix}(F, \sigma)$ is separable. If finally $(\Omega, \sigma)$ is a model of $S C F A_{e}$, then the $p$-basis $\bar{b}$ is fixed by $\sigma$, and is hence a $p$-basis of $\operatorname{Fix}(\Omega, \sigma)$.

Proof. We may assume that our difference field, call it again $(F, \sigma)$, is separably closed by lemma (5.1). We choose a $p$-basis $\bar{b}=b_{1}, \ldots, b_{e}$ of $k$. then of course $\bar{b}$ is also a $p$-basis of $k^{\text {sep }}$, and we expand $k^{\text {sep }}$ to an $L$-structure. As mentioned above, $\bar{b}$ is $p$-independent in $F$.

Now lemma $(2.2)$ of [3] states that if $(K, \sigma)$ is a difference field with finite $p$ basis $B$ and $K(\bar{a})_{\sigma}$ is a finitely generated separable difference field extension of $(K, \sigma)$, then $K(\bar{a})_{\sigma}$ embeds into some difference field with $p$-basis $B$. This implies that for any finite tuple $\bar{a} \in F$, the difference field $k^{\operatorname{sep}}(\bar{a})_{\sigma}$ embeds into some separably closed difference field with $p$-basis $\bar{b}$, or in other words into some model of $\left(S C F_{e, b}\right)_{\sigma}$, as $\bar{b}$ is fixed by $\sigma$. Thus by compactness $(F, \sigma)$ embeds into some model of $\left(S C F_{e, b}\right)_{\sigma}$, and consequently into some model $(\Omega, \sigma)$ of $S C F A_{e, b}$ by model completeness.

By the above remarks, $\bar{b}$ is a $p$-basis of $\operatorname{Fix}(\Omega, \sigma)$, whence $\operatorname{Fix}(\Omega, \sigma) / k$ is a separable field extension. As $k$ is algebraically closed in $\operatorname{Fix}(\Omega, \sigma), \operatorname{Fix}(\Omega, \sigma) / k$ is a regular extension, and hence elementary by corollary $(20.4 .3)$ of [9]. $\operatorname{Gal}(k)=$ $\widehat{\mathbb{Z}}$ by assumption and $F$ is algebraically closed in the sense in $S C F_{e, b}$, so we apply theorem (4.1) to finish the proof.

Corollary 5.8 Any one-free PAC field with finite Ershov invariant $e$ is isomorphic to the fixed field of some model of $S C F A_{e}$.

Proof. Apply theorem (5.7) to the difference field $(k, \mathrm{id})$.

Note again that any choice of a topological generator of $\mathrm{Gal}(k)$ gives nonelementarily equivalent models of $S C F A_{e}$ with fixed field $k$. 


\section{Elimination of Imaginaries}

In this section we prove a result concerning elimination of imaginaries for $T A$.

Any simple theory satisfies the Independence Theorem over models, see [15]. As Chatzidakis and Pillay show in [6], TA even satisfies the Independence Theorem over models of $T_{\sigma}$, see theorem (3.7) in [6]. We say that $T A$ satisfies the Independence Theorem over algebraically closed sets if it satisfies the Independence Theorem over algebraically closed sets of the home sort.

Proposition 6.1 Let $T$ be stable and eliminate imaginaries and let $T A$ satisfy the Independence Theorem over algebraically closed sets. Then TA eliminates imaginaries (i.e. every completion of TA does).

The statement is proved for example when $T$ is $A C F$ by Chatzidakis and Hrushovski in [4] and for strongly minimal $T$ with $\operatorname{acl}_{T}(\emptyset)$ infinite by Chatzidakis and Pillay in [6]. We step here along Pillays proof line in [12] where he did the strongly minimal case.

Proof. Let $(M, \sigma)$ be a model of $T A$ and $e$ be an imaginary element. We may assume that $(M, \sigma)$ is sufficiently saturated. To distinguish, we write $\operatorname{acl}_{\sigma}$ and $\mathrm{dcl}_{\sigma}$ if we compute the respective closure in the home sort, and acl $l_{\sigma}^{\mathrm{eq}}$ and $\mathrm{dcl}_{\sigma}^{\mathrm{eq}}$ if we compute the respective closure in $(M, \sigma)^{\text {eq }}$. Choose a $\emptyset$-definable function $f$ and a tuple $\bar{a} \in M$ such that $e=f(\bar{a})$. Let $\bar{b}$ realise the type of $\bar{a}$ over $e$ and be independent from $\bar{a}$ over $e$. Further let $\bar{c} \in M$ realise the type of $\bar{a}$ over $e$ and be independent from $\operatorname{acl}_{\sigma}(\bar{a}, \bar{b})$ over $\operatorname{acl}_{\sigma}^{\mathrm{eq}}(e)$.

Because $T$ is stable and eliminates imaginaries, there is a unique smallest acl $_{\sigma^{-}}$ closed subset $A \subset M$ (not $M^{e q}$ ) such that $c$ is independent from $\operatorname{acl}_{\sigma}^{e q}(\bar{a}, \bar{b})$ over $A$. Namely, take for $A$ the $\operatorname{acl}_{T}$-closure of the canonical base of $p=$ $\operatorname{tp}\left(\sigma^{i}(c)_{i \in \mathbb{Z}} / \operatorname{acl}_{\sigma}^{\mathrm{eq}}(a)\right)$, all in the sense of $T$. Clearly $\sigma(p)=p$, hence $A$ is invariant under $\sigma$ and $\sigma^{-1}$ and thus it is $\operatorname{acl}_{\sigma}$-closed by fact (2.4.2). By the definition of independence in $T A$, see $(2.4 .3)$, one sees that $A$ is smallest possible.

By transitivity we have $A \subseteq \operatorname{acl}_{\sigma}(\bar{a})$ and $A \subseteq \operatorname{acl}_{\sigma}(\bar{b})$, whence we get that $A \subseteq$ $\operatorname{acl}_{\sigma}(\bar{a}) \cap \operatorname{acl}_{\sigma}(\bar{b}) . \operatorname{As~acl}_{\sigma}^{\mathrm{eq}}(\bar{a}) \cap \operatorname{acl}_{\sigma}^{\mathrm{eq}}(\bar{b})=\operatorname{acl}_{\sigma}^{\mathrm{eq}}(e)$ we conclude that $A \subseteq \operatorname{acl}_{\sigma}^{\mathrm{eq}}(e)$. Since $c$ is independent from $\operatorname{acl}_{\sigma}^{\mathrm{eq}}(\bar{a}, \bar{b})$ it follows again by transitivity that $c$ is independent from $e$ over $A$, and thus that $e$ is independent from $c$ over $A$. But $e$ is algebraic over $c$, so it must be algebraic already over $A$.

We want to show that $e$ is even definable over $A$. To that end choose any realisation $\bar{a}^{\prime}$ of $\operatorname{tp}_{\sigma}(\bar{a} / A)$. There is some $\bar{b}^{\prime}$ realising $\operatorname{tp}_{\sigma}\left(\bar{a}^{\prime} / A\right)$ which is independent from $\bar{a}^{\prime}$ over $A$, with $f\left(\bar{b}^{\prime}\right)=f\left(\bar{a}^{\prime}\right)$ and which is independent from $\bar{b}$ over $A$. As $A$ is acl $_{\sigma}$-closed we can apply the Indepedence Theorem over algebraically closed sets to find some realization $\bar{d}$ of $\operatorname{tp}_{\sigma}(\bar{a} / A \bar{b}) \cup \operatorname{tp}_{\sigma}\left(\bar{a}^{\prime} / A \bar{b}^{\prime}\right)$. But then $f(\bar{a})=f(\bar{d})=f\left(\bar{a}^{\prime}\right)$, so it follows that $e$ is definable over $A$.

What we have shown is that there is some real tuple $\bar{a}^{\prime}$ with $\bar{a}^{\prime} \in \operatorname{acl}_{\sigma}^{e q}(e)$ and $e \in \operatorname{dcl}_{\sigma}^{e q}\left(\bar{a}^{\prime}\right)$. As $T$ eliminates imaginaries, the set of $e$-conjugates of $\bar{a}^{\prime}$ is interdefinable with a real tuple, and thus so is $e$. 


\section{References}

[1] James Ax: The Elementary Theory of Finite Fields. Annals of Math. 88 (1968), 239-271.

[2] Ronald Bustamante-Medina: Differentially Closed Fields of Characteristic zero with a Generic Automorphism. Revista de Matemática: Teoría y Aplicaciones 2007 14(1), 81-100.

[3] Zoé Chatzidakis: Generic Automorphisms of Separably Closed Fields. Illinois Journal of Mathematics. Volume 45, Number 3, Fall 2001, 693-733.

[4] Zoé Chatzidakis, Ehud Hrushovski: Model Theory of Difference Fields. Trans. Amer. Math. Soc. 351 (1999), 2997-3071.

[5] Zoé Chatzidakis, Ehud Hrushovski, Ya'acov Peterzil: Model Theory of Difference Fields, II: Periodic Ideals and the Trichotomy in all Characteristics. Proceedings of the London Mathematical Society (3) 85 (2002) 257-311.

[6] Zoé Chatzidakis, Anand Pillay: Generic Structures and Simple Theories. Annals of Pure and Applied Logic 95 (1998), 71-92.

[7] Richard M. Cohn: Difference Algebra. Tracts in Mathematics 17, Interscience Pub. 1965.

[8] Francoise Delon: Idéaux et Types sur les Corps séparablement clos. Mém. Soc. Math. France (N.S.) 33 (1988), 1-76.

[9] Michael Fried, Moshe Jarden: Field Arithmetic. Springer, 2nd edition 2005.

[10] Serge Lang: Algebra. Fourth Edition, Springer 2002.

[11] Hideyuki Matsumura: Commutative Ring Theory. Cambridge University Press.

[12] Anand Pillay: Strongly Minimal Sets with a Generic Automorphism. Lecture Notes 2005.

[13] Anand Pillay, Dominika Polkowska: On PAC and Bounded Substructures of a Stable Structure. Journal of Symbolic Logic 71 (2006), 460-472.

[14] Bruno Poizat: Theorie de Galois Imaginaire. Journal of Symbolic Logic 48 (1983) 1151-1170.

[15] Frank O. Wagner: Simple Theories. Kluwer Academic Publishers 2000.

[16] Martin Ziegler: Stabilitätstheorie. Vorlesungsskript 1989, available at http://home.mathematik.uni-freiburg.de/ziegler/ 